\title{
Failure to Adequately Clean
}

National Cancer Institute

\section{Source}

National Cancer Institute. Failure to Adequately Clean. NCI Thesaurus. Code C133637.

Problem associated with the failure of the device or operator to remove any visible soil, foreign material or organism deposits on the external surfaces, crevices, and joints of the device. 